\title{
85 DETECTION OF HUMAN ANGIOTENSIN-CONVERTING ENZYME 2 RECEPTOR (HACE2R) ON HUMAN CANCER CELL LINES
}

Tarsem Moudgil*, Bernard Fox, Hong-Ming Hu. E.A.Chiles Research INST, Portland, OR, USA

Background SARS-CoV-2 infections have delayed administration of treatments for some patients with cancer, increasing the number of avoidable deaths. However, we hypothesized that infection of cancer cells with SARS-CoV-2 might increase the immunogenicity of those cancer cells. Here we sought to determine whether non-small cell lung cancer (NSCLC) and head and neck squamous cell cancer (HNSCC) cell lines could be a potential target of SARS-CoV-2, which binds and infects host cells via interactions between the viral spike glycoprotein and the human angiotensin-converting enzyme 2 receptor (hACE2) receptor. Through an institutional research tissue protocol, our lab has established a panel of cancer cell lines of various histologies. Here we set out to identify whether HNSCC and NSCLC cell lines expressed the hACE2R. We also investigated the expression of neuropilin-1, a molecule reported to facilitate SARS-CoV-2 cell entry.

Methods Established cell lines were phenotyped by flow cytometric analysis utilizing the anti-hACE2R antibody from Novus Biologicals. Cell lines were also stained with mIgG1 and anti-CD3 antibodies as negative staining controls.

Results We identified that three of eight NSCLC cell lines expressed the hACE2R and two of these had strong expression of neuropilin-1. Evaluation of HNSCC cell lines identified seven of seven cell lines expressed detectable levels of hACE2R but only one of seven HNSCC celllines expressed substantial levels of neuropilin-1. Preliminary evaluation of a renal cell carcinoma (RCC) cell line revealed strong staining for hACE2R.

Conclusions Our study found that a majority of HNSCC cell lines $(100 \% \mathrm{n}=7)$ and approximately a third of the NSCLC cell lines $(37.5 \%, \mathrm{n}=8)$ tested express the hACE2R. Some cell lines express both hACE2R and neuropilin-1, potentially increasing their susceptibility for infection with SARS-C0V-2. While these studies were performed with cultured cell lines that may have modulated expression of hACE2R, it is possible that in vivo these tumors express the ACE2R and could serve as a target and possible reservoir for SARS-CoV-2.

Acknowledgements

Support The Chiles foundation, Nancy Lematta

http://dx.doi.org/10.1136/jitc-2021-SITC2021.085 